\title{
Procedimento de Resposta de Observação e Comportamento de Observação
}

\section{Observing Respose Design and Observing Behavior \\ Delineamiento de Respuesta de Observación y Comportamiento de Observación}

\author{
Candido V. B. B. Pessôa ${ }^{1}$, Gerson Y. Tomanari²
}

[1] Paradigma - Centro de Ciências do Comportamento [2] Instituto de Psicologia da Universidade de São Paulo | Título abreviado: Comportamento e Procedimento de Observação I Endereço para correspondência: Associação Paradigma - Centro de Ciências e Tecnologia do Comportamento, Rua Vanderley, 611, Perdizes, São Paulo/SP I Email: candidopessoa@uol.com.br I DOI: 10.18761/pac.0214

Resumo: A expressão resposta de observação foi criada por Wyckoff (1952) para indicar respostas que produzem estímulos discriminativos para outras respostas. A análise dessas respostas esclarece como se dá a discriminação em situações nas quais a exposição aos eventos do ambiente que eventualmente se tornarão estímulos discriminativos não é garantida $a$ priori. Os Estudos de Wyckoff possibilitaram a ampliação das teorias de discriminação baseadas na ação do ambiente sobre os indivíduos. Posteriormente, o procedimento utilizado pelo autor, conhecido por procedimento de resposta de observação, foi grandemente utilizado em pesquisas sobre a origem da função de reforçador condicionado. Os requisitos desse procedimento não podem ser confundidos com o comportamento de observação, sob pena de dificultar o estudo sobre a instalação do comportamento de observação em situações práticas.

Palavras-chave: resposta de observação; procedimento de resposta de observação; comportamento de observação; atenção

\begin{abstract}
The expression observing responses was created by Wyckoff (1952) to indicate responses that produce discriminative stimuli for other responses. The analysis of these responses clarifies how discrimination occurs in situations that the exposure to environmental events that may become discriminative stimuli is not assured a priori. Wyckoff's studies enabled the extension of theories of discrimination based on the action of the environment on individuals to such cases. Subsequently, the design used by the author, known as observing response design, was largely used in research on the origin of the conditioned reinforcer stimulus function. The design requirements cannot be confused with the observing behavior itself, otherwise precluding studies on the installation of behavior observation in practical situations.
\end{abstract}

Keywords: observing response; observing response design; observing behavior; attention. 
Resumen: La expresión respuesta de observación fue creada por Wyckoff (1952) para indicar respuestas que producen estímulos discriminadores para otras respuestas. El análisis de esas respuestas aclara cómo se da la discriminación en situaciones en las que la exposición a los eventos del ambiente que eventualmente se tornarán estímulos discriminadores no está garantizada a priori. Los estudios de Wyckoff permitieron la ampliación de las teorías de discriminación basadas en la acción del ambiente sobre los individuos. Posteriormente, el delineamiento utilizado por el autor, conocido como delineamiento de respuesta de observación, fue ampliamente utilizado en investigaciones sobre el origen de la función de reforzador condicionado. Los requisitos de este delineamiento no se pueden confundir con el comportamiento de observación, con el riesgo de dificultar el estudio sobre la instalación del comportamiento de observación en situaciones prácticas.

Palabras-clave: respuesta de observación; delineamiento de respuesta de observación; comportamiento de observación; atención. 
O objetivo deste artigo é diferenciar as expressões "comportamento de observação" e "procedimento de resposta de observação", indicando a que se refere cada uma dessas expressões na análise do comportamento. Intui-se que essa diferenciação possa facilitar o desenvolvimento de pesquisas que visem à melhoria de técnicas de instalação de comportamento de observação eficaz, assim como ao desenvolvimento de um maior número de pesquisas translacionais sobre o assunto.

\section{Contexto Histórico}

A expressão resposta de observação surge no contexto de investigação da aprendizagem da discriminação. A partir da década de 1930, uma controvérsia que ficou conhecida pelo nome de "continuidade ou descontinuidade" instalou-se nessa área. Spence (1940) sintetiza a controvérsia da seguinte maneira: a hipótese da continuidade, baseada em conceitos de associação e de interações entre estímulos e respostas, explicaria a discriminação como um contínuo de acúmulo de pequenas diferenças no valor dos estímulos envolvidos em uma discriminação até que alguns desses estímulos adquirissem funções discriminativas e outros se tornasses irrelevantes; pela hipótese da descontinuidade, a discriminação ocorreria de maneira discreta, baseada em um repertório de hipóteses do sujeito, testadas até descobrir-se o aspecto relevante do ambiente para o controle discriminativo. Ao longo das décadas de 1930 e 1950, dados experimentais surgiram de forma a fortalecer e enfraquecer ambos os lados da controvérsia (para revisão dos dados existentes à época, veja Bitterman \& Coate, 1950).

Wyckoff (1952) apontou um dos possíveis fatos a gerar dados contrários à hipótese da continuidade: as teorias que formavam a visão da continuidade (e.g., Skinner, 1935/1999c, 1937/1999c, 1938/1991; Spence, 1936, 1937) apresentavam a discriminação em situações nas quais procurava-se garantir o contato dos órgãos sensoriais do organismo com os estímulos relevantes à discriminação. (Essa situação satisfaz a ideia da tríplice contingência como uma unidade de seleção.) Porém, como afirma Wyckoff, mesmo em situações controladas de laboratório, nunca ou raramente é possível afirmar-se que o sujeito foi inquestionavelmente exposto aos estímulos relevantes antes da resposta que produz o reforço ocorrer. Essa falta de controle experimental explicaria, primeiramente, parte da variabilidade nos dados obtidos nos experimentos até então e também constituiria um limite às teorias de discriminação condizentes com a hipótese da continuidade.

Diante das considerações feitas, Wyckoff (1952) decide estudar a relevância de uma classe de respostas externa à tríplice contingência na aprendizagem da discriminação: as respostas que produzem os estímulos discriminativos para outras respostas. Wyckoff nomeia essas respostas de respostas de observação (ROs).

\section{Os Objetivos de Wyckoff}

Referindo-se a Skinner (1938/1991), Wyckoff (1952) estabelece como hipótese geral de sua pesquisa que a exposição a um par de estímulos discriminativos teria efeito reforçador sobre as ROs na extensão em que o sujeito tenha aprendido a responder diferencialmente ao par de estímulos. Como hipóteses específicas, Wyckoff propõe que: (a) a probabilidade de emissão das ROs aumenta ou permanece alta sob condições de reforço diferencial, (b) a probabilidade diminui ou permanece baixa em situações de reforço não diferencial; (c) quando uma discriminação bem estabelecida é revertida, a probabilidade de emissão das ROs medidas vai decrescer temporariamente e depois retornar a um valor alto; e (d) se em algum ponto do experimento o grau de discriminação - i.e., desempenho diferencial diante de cada estímulo do par - for baixo porém maior que zero, espera-se que a formação da discriminação seja retardada por um tempo e depois aconteça rapidamente.

\section{O Experimento de Wyckoff}

Para testar as hipóteses propostas, Wyckoff (1969) ${ }^{1}$ realizou um experimento em que foram utilizados 20 pombos em privação de alimento. Durante as sessões, cada pombo foi colocado em uma caixa experimental contendo um disco que podia ser iluminado de branco, vermelho ou verde, um comedouro retrátil e

1 A data de 1969 se refere à publicação dos dados obtidos por Wyckoff em sua tese de doutorado, The role of observing responses in discrimination learning: Part II, apresentada em 1951 na Indiana University. 
um pedal. O procedimento de Wyckoff consistiu-se de dois grupos de sujeitos e duas fases experimentais.

A primeira fase (Fase Preliminar) durou $45 \mathrm{mi}-$ nutos. Durante os primeiros 15 minutos o disco foi iluminado de branco. A primeira bicada no disco após decorridos $30 \mathrm{~s}$ da apresentação da iluminação ou do último recebimento de comida produzia acesso ao comedouro por quatro segundos e o apagamento da luz branca (esquema de intervalo fixo de $30 \mathrm{se}$ gundos - FI 30). Após os quatro segundos de acesso ao comedouro, o disco voltava a ser iluminado com a luz branca. Durante os 30 minutos restantes dessa fase, o disco foi iluminado de verde ou de vermelho em componentes de 30 segundos, apresentados sucessivamente em ordem semi-aleatória. As respostas de bicar o disco foram reforçadas com quatro segundos de acesso ao comedouro em FI 30 em metade dos componentes em que o disco foi iluminado de verde e em metade dos componentes em que o disco foi iluminado de vermelho. Durante essa fase não houve contingência programada para pressões no pedal; porém, essas respostas foram registradas.

$\mathrm{Na}$ segunda fase (Fase Experimental), primeiramente houve cinco sessões de 75 minutos e uma de 15 minutos. Durante essas sessões, componentes de 30 segundos que poderiam acabar em reforço (em FI 30, a depender da emissão de bicadas no disco) se alternaram de forma semi-aleatória e sem intervalo com componentes de 30 segundos nos quais não havia reforço programado para bicadas no disco (extinção - EXT). É importante salientar que nada que o pombo fizesse podia alterar a ordem ou a duração dos componentes ou o número de acessos programados ao comedouro. Durante as sessões dessa fase o disco foi iluminado de branco, a não ser que o pombo emitisse a resposta de pisar no pedal. Durante o tempo em que o pedal estivesse pressionado o disco era iluminado de vermelho ou verde pelo tempo que durasse a resposta ou até o fim do componente em vigor.

Para testar as hipóteses (a) e (b), no início da Fase Experimental, os pombos foram divididos em dois grupos. Para o Grupo I (controle), com quatro pombos, as cores produzidas pelas pressões nos pedais, vermelho e verde, não tinham relação consistente com a presença ou não de reforço no fim do componente. Para o Grupo II (experimental), com 16 pombos, o disco iluminado de vermelho foi consistentemente relacionado aos componentes em que podia haver reforço e o disco iluminado de verde foi relacionado aos componentes de EXT. Para esse grupo, então, pressões no pedal podiam equivaler às ROs, pois permitiam o contato com os estímulos discriminativos para a tarefa (Wyckoff, 1969). Foram registradas as frequências das bicadas diante de cada estímulo (verde e vermelho), separadamente, e as durações das pressões no pedal de cada sessão de cada pombo.

Após os 15 minutos da sexta sessão da Fase Experimental, os 16 pombos do Grupo II (experimental) foram divididos em três subgrupos. Os pombos do Grupo II-a $(\mathrm{n}=6)$ foram submetidos a mais sete sessões de reforço diferencial tal como já vinha ocorrendo na Fase Experimental (completaram mais 60 minutos restantes da sexta sessão e cumpriram mais seis sessões de 75 minutos). Os pombos do Grupo II-b $(n=5)$ foram submetidos a sete sessões de reforço diferencial com a discriminação revertida. Para esse grupo, assim como para o seguinte, a situação mudou após os 15 minutos da última sessão da fase anterior sem interrupção da sessão. Os pombos do Grupo II-c $(n=5)$ foram submetidos a sete sessões de reforço não diferencial. A Tabela 1 sintetiza as condições experimentais de cada grupo na Fase Experimental.

\section{Tabela 1. Condições experimentais de cada grupo de participantes na Fase Experimental do experimento de Wyckoff (adaptado de Wyckoff, 1969)}

\begin{tabular}{|c|c|c|}
\hline \multirow[b]{2}{*}{ Grupo } & \multicolumn{2}{|c|}{ Sessões } \\
\hline & $1-6^{*}$ & $6^{*}-12$ \\
\hline $\begin{array}{c}\text { I } \\
\text { (sem } \\
\text { discriminação) } \\
\mathrm{n}=4\end{array}$ & $\begin{array}{l}\text { reforçamento } \\
\text { não diferencial }\end{array}$ & $\begin{array}{c}\text { reforçamento não } \\
\text { diferencial }\end{array}$ \\
\hline $\begin{array}{c}\| \text { (discriminação) } \\
n=6\end{array}$ & $\begin{array}{c}\text { reforçamento } \\
\text { diferencial }\end{array}$ & $\begin{array}{c}\text { reforçamento } \\
\text { diferencial } \\
\text { continuado }\end{array}$ \\
\hline $\begin{array}{c}\| l-b \\
\text { (reversão da } \\
\text { discriminação) } \\
n=6\end{array}$ & $\begin{array}{c}\text { reforçamento } \\
\text { diferencial }\end{array}$ & $\begin{array}{l}\text { reforçamento } \\
\text { diferencial com } \\
\text { reversão da } \\
\text { discriminação }\end{array}$ \\
\hline $\begin{array}{c}\| l-c \\
\text { (abolição da } \\
\text { discriminação) } \\
n=6\end{array}$ & $\begin{array}{c}\text { reforçamento } \\
\text { diferencial }\end{array}$ & $\begin{array}{c}\text { reforçamento não } \\
\text { diferencial }\end{array}$ \\
\hline
\end{tabular}

6* denota o fim dos primeiros quinze minutos da sessão 6 
Como resultado, verificou-se que os pombos do Grupo II (experimental) aumentaram a proporção de tempo pressionando o pedal na Fase Experimental em relação à Fase Preliminar e passaram a bicar o disco quando vermelho era produzido e a não bicá-lo quando verde era produzido. Já para os pombos do Grupo I (controle), a proporção de tempo pressionando-se o pedal na Fase Experimental diminuiu em relação à primeira fase e não houve responder discriminado das respostas de bicar o disco em relação às cores vermelho e verde na Fase Experimental. Esses resultados confirmaram as hipóteses (a) e (b) feitas por Wyckoff (1952). De acordo com a hipótese (c), era esperado que para os pombos do Grupo II-b o comportamento de observação diminuísse temporariamente quando houvesse a reversão e que depois esse comportamento recuperasse seu valor. Esse foi o desempenho verificado, confirmando a hipótese (c). A hipótese (d) apenas poderia ser verificada se em algum ponto das sessões a discriminação estivesse fracamente estabelecida e a probabilidade de emissão das ROs também fosse baixa. Isso ocorreu, segundo Wyckoff (1969), com alguns pombos do Grupo II durante as primeiras seis sessões e também com a maioria dos pombos do Grupo II-b logo após a reversão da discriminação, confirmando a hipótese (d). Finalmente, após a abolição da discriminação ocorrida no Grupo II-c, o tempo de emissão das ROs diminuiu em relação ao tempo de emissão antes da abolição da discriminação. Esses resultados permitem afirmar, tendo os sujeitos como seus próprios controles, que o comportamento de observação foi selecionado (i.e., gerado e mantido) pela produção dos estímulos discriminativos.

Os resultados apresentados permitiram que Wyckoff $(1952,1969)$ atingisse seu objetivo de ampliar a generalidade das teorias de discriminação baseadas na ação do ambiente sobre as respostas para situações em que repostas de observação são requeridas para haver exposição aos estímulos discriminativos. Tendo os resultados de Wyckoff como base, pode-se afirmar, então, que a ocorrência de discriminações envolve a análise de dois comportamentos, o comportamento de observação e o comportamento de produção ${ }^{2}$ (Pessôa \& Sério,

2 O nome comportamento de produção é dado apenas como forma de distinguir os dois comportamentos envolvidos nessa
2006). Ainda, é importante notar, como destacam Dinsmoor, Flint, Smith e Viemeister (1969), que os estímulos discriminativos produzidos pelas ROs no experimento de Wyckoff fazem parte de dois processos comportamentais ${ }^{3}$ : reforçamento condicionado (da resposta de observação) e estabelecimento do controle por estímulos discriminativos ou discriminação (no comportamento de produção).

\section{Utilizações do Procedimento Experimental de Resposta de Observação de Wyckoff na Pesquisa Básica}

Wyckoff $(1952,1969)$ é reconhecido pela engenhosidade com que conseguiu facilitar o registro de uma resposta que fora do laboratório é muito difícil de ser registrada. Ao usar o pedal como resposta de observação, Wyckoff emulou topografias de resposta que são muito sutis para serem registradas, tais como o movimento dos olhos ou mesmo respostas que ainda não se tem como registrar, como o caso de controles auditivos em que um indivíduo observa o som do violino em um quarteto de cordas.

Uma segunda engenhosidade do procedimento utilizado foi muito apreciada na pesquisa básica da análise experimental do comportamento. A emissão de uma resposta de observação que transfor-

situação, pois no comportamento de observação os estímulos discriminativos são, também, produzidos. Comportamento de produção - e instâncias da classe de respostas deste comportamento, resposta de produção - é, aqui, arbitrariamente utilizado por razões apresentadas em Pessôa e Sério (2006). Dentre outros nomes que são utilizados para se referir a este comportamento - ou suas respostas - controlado pelos estímulos produzidos pelo comportamento de observação estão: respostas alvo (Spence, 1936, 1937); respostas efetivas (Wyckoff, 1952, 1969); respostas discriminadas (Dinsmoor, 1983, 1985); respostas principais (Pergher, 2007; Tomanari, 2008); e comportamento corrente (Strapasson \& Dittrich, 2008). Como pode ser discutido, todos os adjetivos usados para especificar este segundo comportamento poderiam especificar o comportamento de observação.

3 Processo é uma palavra que comporta diversas interpretações. No presente trabalho, a expressão "processo comportamental" é usada significando mudanças no comportamento (Skinner, 1968/2003b, p. 120). Essas mudanças são devidas a operações tais como reforço (apresentação de um reforçador contingente a uma resposta) e extinção (suspensão do reforço). 
ma momentaneamente um esquema misto ${ }^{4} \mathrm{em}$ um esquema múltiplo ${ }^{5}$ constituiu-se como uma ótima opção ao uso de esquemas encadeados no estudo da aquisição da função reforçadora condicionada. Em esquemas encadeados, a produção do reforçador no último elo da cadeia depende da produção de cada estímulo intermediário (Ferster \& Skinner, 1957). Por exemplo, a resposta de um rato passar por dentro de uma argola produz luz azul e apenas diante dessa luz a resposta de pressão à barra produz uma gota de água no bebedouro. Nesses casos, o efeito reforçador do estímulo discriminativo (luz azul) sobre a resposta que o produz (passar pela argola) pode se confundir com o efeito reforçador do estímulo no último elo da cadeia (água) sobre essa mesma resposta (passar pela argola). Já no procedimento proposto por Wyckoff, a relação entre o estímulo produzido pela $\mathrm{RO}$ e o reforçador do comportamento de produção não é de contingência. A produção do reforçador no comportamento de produção não depende da emissão das ROs especificadas. Note-se que no experimento relatado os pombos poderiam produzir alimento tanto diante do estímulo distintivo dos componentes de FI (luz vermelha) como em sua ausência (luz branca). O procedimento de Wyckoff (1969), em que a RO produz estímulos discriminativos para um operante sem alterar a programação de reforço desse operante, passou a ser conhecido como procedimento de resposta de observação (e. g. Dinsmoor, 1983, 1985, 1995; Gollub, 1970; Hirota, 1972, 1974). (Para se entender a diferença entre o procedimento de resposta de observação aqui discutido e o procedimento usado em experimentos envolvento matching to sample sugerimos a leitura de Pessôa e Sério, [2006].)

No exemplo fornecido pelo experimento de Wyckoff (1969), o pombo poderia receber exatamente a mesma quantidade de alimento emitindo ou não a RO. Essa dupla possibilidade de curso de ação adiciona, sem dúvida, complexidade à análi-

4 i.e., dois ou mais esquemas simples intercalados em componentes sucessivos sem estímulos que os discriminem (Ferster \& Skinner, 1957)

5 i.e., dois ou mais esquemas simples intercalados em componentes sucessivos com estímulos que os discriminem (Ferster \& Skinner, 1957). se do comportamento. Por que o pombo emitiria o comportamento de observação? A relativa independência entre o valor reforçador dos estímulos que mantêm o comportamento de observação e o valor reforçador dos estímulos que mantêm o comportamento de produção no procedimento de resposta de observação favoreceu a utilização desse procedimento em grande quantidade de pesquisas que investigaram a origem e a manutenção da função reforçadora condicionada. Os achados dessas pesquisas não são objeto deste texto, mas as principais contribuições no refinamento do conceito de reforçamento condicionado que empregam este procedimento são constantemente revisadas (e.g., Dinsmoor, 1983; Fantino, 1977, 2001; Fantino \& Silberberg, 2010; Shahan, 2010; Tomanari, 2000, 2008; Williams, 1994 ).

Nos estudos sobre a origem da função reforçadora condicionada que utilizam procedimentos de resposta de observação a variável dependente medida é alguma dimensão da resposta de observação em dois momentos diferentes (geralmente, antes e depois do estabelecimento da discriminação). Quanto mais o fortalecimento das ROs pela produção de estímulos discriminativos na contingência de produção for independente do reforço do comportamento de produção, mais estará assegurado que o fortalecimento do comportamento de observação se deu somente pela produção dos estímulos discriminativos.

\section{A Importância do Comportamento de Observação}

A importância do comportamento de observação (para a diferença entre resposta de observação e comportamento de observação consulte Pessôa e Sério [2006].) não se exaure na utilização do procedimento de resposta de observação. Wyckoff (1952, p.432) realça a importância das situações em que uma resposta de observação é requerida para o estabelecimento de discriminações em situações cotidianas como o primeiro motivo de estudá-las. Além de Wyckoff, outro autor que ressalta a importância de pesquisas sobre o comportamento de observação é B. F. Skinner, principalmente no contexto da educação (Skinner 1954/2003a, 1957/1992, 1958/1999a, 1961/1999d, 1968/2003b). Mas, antes 
de aprofundar esse assunto, talvez valha entender como Skinner recoloca a definição de comportamento de observação.

Retorne-se à definição de RO apresentada no início do texto: respostas de observação são aquelas que produzem estímulos discriminativos para outras respostas. Se olhada apenas nestes termos, sem o requisito imposto por Wyckoff em seu procedimento, qualquer resposta em elo de um esquema encadeado se constituiria como uma resposta de observação, tornando-se assim extremamente ampla a definição da resposta. Talvez por isso o cuidado de Skinner (1954/2003a) ao apresentar o conceito:

É possível, também, construir sequências bem complexas de esquemas. Não é fácil descrevê-las em poucas palavras, mas dois ou três exemplos podem ser mencionados. Em um experimento, o pombo gera um desempenho apropriado ao Esquema A, no qual o reforço é simplesmente a produção do estímulo característico do Esquema B, ao qual o pombo responde apropriadamente. Sob um terceiro estímulo, a ave realiza um desempenho apropriado ao Esquema $\mathrm{C}$ no qual o reforço é simplesmente a produção dos estímulos característicos do Esquema D, ao qual a ave então responde apropriadamente [produzindo acesso a comida]. Em um caso especial, primeiro investigado por L. B. Wyckoff, Jr., o organismo responde a um estímulo no qual o reforço consiste na clarificação do estímulo controlando outra resposta. A primeira resposta se torna, por assim dizer, uma forma objetiva de 'prestar atenção' no segundo estímulo.” (p. 13, grifos no original).

A citação de Skinner (1954/2003a) a Wyckoff (1952) foi feita em um contexto de explanação sobre "a ciência da aprendizagem e a arte do ensino". Nela podemos perceber que Skinner (a) diferencia a resposta de observação de um elo em um esquema encadeado, (b) colocando a clarificação do estímulo controlador do comportamento de produção como o reforçador do comportamento de observação.

No caso de (a), Skinner usa a expressão "sequências bem complexas de esquemas". O termo "esquemas" se refere a arranjos de relações entre os termos da tríplice contingência. Esses arranjos causam desempenhos típicos e diferentes entre si. Por exemplo, o desempenho em meia lua (em inglês referido como scallop) característico de esquemas em intervalo fixo, ou o desempenho bi-tônico (em inglês referido como stop-and-run) característico de esquemas em razão fixa. "Sequências complexas" indica que mais de uma tríplice contingência está em questão. Na sequência da citação há um exemplo do esquema que logo depois será denominado como esquema encadeado (Ferster \& Skinner, 1957). Em esquemas encadeados mais de uma condição tem que ser satisfeita para se obter o reforço (o raciocínio lógico aqui é simplificado, falando-se de reforço apenas o caso de produção de um reforçador incondicionado e não de reforçadores condicionados intermediários criados exatamente por conta deste último). No exemplo de Skinner, a única forma de o pombo receber o alimento é passando por todos os esquemas e produzindo todos os estímulos intermediários. O que torna o caso do comportamento de observação especial é o fato de a $\mathrm{RO}$ não precisar necessariamente ser emitida para a obtenção do reforço. "Clarificar o estímulo controlador" é diferente de "produzir o estimulo controlador”. À primeira vista, ao ler-se a definição de Wyckoff (1952) pode-se ter a impressão de que sem a emissão da RO o indivíduo estaria se comportando sem estímulos discriminativos. Mas, deve-se lembrar que dificilmente um indivíduo se comporta sem a presença de um SD (Skinner, 1938/1991). No caso de Wyckoff (1969), sem a emissão da RO o estímulo discriminativo para a emissão da resposta de produção seria a luz branca. Assim, como dito anteriormente, no caso do comportamento de observação, o indivíduo tem dois cursos de ação, um observando e outro sem observar.

A observação não ser imprescindível não quer dizer que ela não altere o ambiente. Novamente, no caso do experimento de Wyckoff, a emissão das ROs fez com que os pombos obtivessem a mesma quantidade de alimento emitindo aproximadamente metade das respostas de produção. Falando de outra forma, Fantino (1977) destaca que o comportamento de observação deu aos pombos acesso a um ambiente com densidade de reforço - ou seja, quantidade de reforços por 
unidade de tempo - duas vezes maior do que o ambiente existente sem a emissão das ROs. Num caso prático, imagine um estudante que lê parte do enunciados das questões de uma prova e assim emite respostas que produzam uma nota medíocre, que talvez leve à necessidade da realização de outra prova para o estudante passar de ano. $\mathrm{O}$ mesmo estudante poderia ler o enunciado todo e emitir uma resposta que produzisse a nota máxima. O comportamento de observação nesse caso seria ler o enunciado todo, o que daria acesso a um reforço de maior magnitude. Porém, ler apenas parcialmente o enunciado também produziria reforço. Possivelmente esse é o sentido do termo clarificar: possibilitar a emissão de uma resposta de produção mais eficiente. Assim, o comportamento de observação é reforçado pela clarificação do estímulo controlador de outra resposta.

Voltando às referências de Skinner sobre o assunto, ao comentar a importância do comportamento de observação na educação (Skinner, 1968/2003b, pp. 121-123), o autor afirma que, no curso normal dos eventos, o reforço que ocorre no comportamento que aqui chamamos de produção acaba por fortalecer o comportamento de observação. Entretanto, deixar isso acontecer pode ser o mesmo que jogar a criança na água para que ela aprenda a nadar: "Simplesmente reforçar uma criança quando ela lê um texto corretamente pode ser muito menos efetivo do que contingências especiais que a induzam a ler da esquerda para a direita ou a ler um bloco de palavras de uma vez." (p. 123). Ressalta-se a importância de não se deixar o comportamento de observação ser reforçado somente pelas suas consequências naturais. Ele deve ser reforçado explicitamente. Em outra passagem Skinner (1961/1999d, p. 237) fornece um exemplo ainda mais dramático da dificuldade que pode haver para o reforçador do comportamento de produção reforçar o comportamento de observação: imagine-se uma criança a quem é mostrada uma figura e, mais tarde, na ausência da figura, reforça-se generosamente respostas corretas a perguntas sobre ela. Se a criança nunca passou por essa experiência antes, ela provavelmente não terá muito sucesso. Se for mostrada, então, uma outra figura a ela, pode ser que ela se engaje nos comportamentos necessários para responder corretamente às ques- tões. Mas será uma vantagem para a criança se for ensinado a ela esses tipos de comportamento em vez de deixá-los ocorrer ao acaso.

As referências de Skinner mostram como, ao menos para esse autor, existe a necessidade de se reforçar o comportamento de observação diretamente e podem surgir problemas por não se fazer isso. $\mathrm{O}$ presente artigo visou diferenciar a utilidade do procedimento de resposta de observação, no qual nunca se deve reforçar diretamente as respostas de observação, da utilidade de um conceito específico de comportamento de observação, ao qual pode ser importante o reforço extrínseco desse comportamento em algumas etapas do aprendizado de um indivíduo. Ainda procurou mostrar-se como o entendimento do comportamento de observação como aquele que clarifica os estímulos controladores de outra resposta pode ajudar a entender a importância prática do comportamento de observação. Considera-se que, entendido assim, o conceito pode ampliar a atuação do analista do comportamento.

\section{Referências}

Bitterman, M. E. \& Coate, W. B. (1950). Some new experiments on the nature of discrimination learning in the rat. Journal of Comparative and Physiological Psychology, 43, 198-210.

Dinsmoor, J. A. (1983). Observing and conditioned reinforcement. Behavioral and Brain Sciences, 6, 693-728.

Dinsmoor, J. A. (1985). The role of observing and attention in establishing stimulus control. Journal of the Experimental Analysis of Behavior, 43, 365-381.

Dinsmoor, J. A. (1995). Stimulus control: part I. The Behavior Analyst, 18, 51-68.

Dinsmoor, J. A., Flint, G. A., Smith, R. F., \& Viemeister, N. F. (1969). Differential reinforcing effects of stimuli associated with the presence or absence of a schedule of punishment. Em D. P. Hendry (Ed.), Conditioned Reinforcement (pp. 357-384). Homewood, IL: Dorsey Press.

Fantino, E. (1977). Conditioned reinforcement: choice and information. Em W. K. Honig \& J. E. R. Staddon (Eds.), Handbook of Operant Behavior (pp. 313-339). Englewood Cliffs, New Jersey: Prentice-Hall, Inc. 
Fantino, E. (2001). Context: a central concept. Behavioural Processes, 54, 95-110.

Fantino, E., \& Silberberg, A. (2010). Revisiting the role of bad news in maintaining human observing behavior. Journal of the Experimental Analysis of Behavior, 93, 157-170.

Ferster, C. B., \& Skinner, B. F. (1957). Schedules of Reinforcement. Englewood Cliffs, New Jersey: Prentice-Hall, Inc.

Gollub, L. R. (1970). Information on conditioned reinforcement: A review of Conditioned Reinforcement, edited by Derek P. Hendry. Journal of the Experimental Analysis of Behavior, 14, 361-372.

Hirota, T. T. (1972). The Wyckoff observing response - a reappraisal. Journal of the Experimental Analysis of Behavior, 18, 263-276.

Hirota, T. T. (1974). The relationship between observing behavior and food-key response rates under mixed and multiple schedules of reinforcement. Journal of the Experimental Analysis of Behavior, 21, 259-266.

Pergher, N. K. (2007). Resposta de Observação em Reversões de Contingências. Tese de doutorado apresentada no Instituto de Psicologia, Universidade de São Paulo, São Paulo, Brasil.

Pessôa, C. V. B. B. \& Sério, T. M. A. P. (2006). Análise do comportamento de observação. Revista Brasileira de Análise do Comportamento, $2,1-12$.

Shahan, T. A. (2010). Conditioned reinforcement and response strength. Journal of the Experimental Analysis of Behavior, 93(2), 269289.

Skinner, B. F. (1991). The Behavior of Organisms. Nova York: Appleton-Century-Crofts. (Publicado originalmente em 1938.)

Skinner, B. F. (1992). Verbal Behavior. Acton, MS: Copley Publishing Group. (Publicado originalmente em 1957.)

Skinner, B. F. (1999a). Teaching Machines. Em B. F. Skinner (Ed.), Cumulative Record: Definitive Edition (pp. 192-216). Acton, MS: Copley Publishing Group. (Publicado originalmente em 1958.)

Skinner, B. F. (1999b). Two types of conditioned reflex: A reply to Konorski and Miller. Em B. F. Skinner (Ed.), Cumulative Record: Definitive
Edition (pp. 525-534). Acton, MS: Copley Publishing Group. (Publicado originalmente em 1937.)

Skinner, B. F. (1999c). Two types of conditioned reflex and a pseudo-type. Em B. F. Skinner (Ed.), Cumulative Record: Definitive Edition (pp. 504524). Acton, MS: Copley Publishing Group. (Publicado originalmente em 1935.)

Skinner, B. F. (1999d). Why we need teaching machines. Em B. F. Skinner (Ed.), Cumulative Record: Definitive Edition (pp. 217-239). Acton, MS: Copley Publishing Group. (Publicado originalmente em 1961.)

Skinner, B. F. (2003a). The science of learning and the art of teaching. Em B. F. Skinner (Ed.), The Technology of Teaching (pp. 9-28). Acton, MS: Copley Publishing Group. (Publicado originalmente em 1954.)

Skinner, B. F. (2003b). The Technology of Teaching. Acton, MS: Copley Publishing Group. (Publicado originalmente em 1968.)

Spence, K. W. (1936). The nature of discrimination learning in animals. Psychological Review, 43, 427-449.

Spence, K. W. (1937). The differential response in animals to stimuli varying within a single dimension. Psychological Review, 44, 430-444

Spence, K. W. (1940). Continuous versus non-continuous interpretations of discrimination learning. Psychological Review, 47, 271-288.

Strapason, B. A., \& Dittrich, A. (2008). O conceito de 'prestar atenção' para Skinner. Psicologia: Teoria e Pesquisa, 24, 519-526.

Tomanari, G. Y. (2000). Reforçamento Condicionado. Revista Brasileira de Terapia Comportamental e Cognitiva, 2, 61-77.

Tomanari, G. Y. (2008). Respostas de Observação: As Principais Questões na Área Analisadas a Partir de Três Experimentos com Pombos. Tese de livre docência apresentada no Instituto de Psicologia, Universidade de São Paulo, São Paulo, Brasil.

Willians, B. A. (1994). Conditioned reinforcement: Experimental and theoretical issues. The Behavior Analyst, 17, 261-285.

Wyckoff, L. B., Jr. (1952). The role of observing responses in discrimination learning: part I. Psychological Review, 59, 431-442. 
Wyckoff, L. B., Jr. (1969). The role of observing responses in discrimination learning. Em D. P. Hendry (Ed.), Conditioned Reinforcement, (pp. 237-260). Homewood: The Dorsey Press.

\section{Informações do Artigo}

Histórico do artigo:

Submetido em: 23/07/2013

Primeira decisão editorial: 25/06/2014

Aceito em: 02/09/2015

Editor Associado: Saulo M. Velasco 